\title{
A Turing Model for Human Cognition
}

\author{
Pooja Asopa ${ }^{1}$, Neelam Sharma ${ }^{2}$ \\ ${ }^{1}$ Banasthali Vidyapith, India, pooja.asopa@gmail.com \\ ${ }^{2}$ Banasthali Vidyapith, India, sharmaneelam27@gmail.com
}

\begin{abstract}
The process of knowledge acquisition deals with the notion of storig the information in long term memory and retrieving the information from long term to short term memory. This process of storing information can be represented in a form of a finite tape. In this research work, a Turing machine model for the information transfer from sensory memory to short term memory is proposed. Along with the model an informal description of an algorithm for information transfer from sensory, to working memory and long term memory is also proposed and discussed. Learning is a continuous process where construction and re-construction of knowledge is reflected in long term memory. The recursive process of learning is influenced by learner's emotional dimension, learning style and its associated external environment.
\end{abstract}

Key words: Cognition, Learning, Long Term Memory, Memory, Sensory Memory, Turing Machine

\section{INTRODUCTION}

Learning a concept is associated with our declarative, procedural and conditional knowledge. How do we perceive any information? How do we learn? Is learning and memory related? How do we convert any information into knowledge? How does information move from sensory memory to working memory? Many researchers, instructional theorists, cognitive psychologists are addressing this quest [1] since decades. The act of learning is an active process where construction and re-construction of knowledge occurs in our Long Term Memory (LTM). Formulation of knowledge, in turn is a process of assimilating information in our LTM, restructuring the information in a form of a cognitive mental model and reflecting on those reconstructed mental models, new knowledge is evolved in a form of a new concept.

Human brain receives simultaneous information through sensory organs, each sensory organ can be considered as a source of information gathering system from the outside world. The information at which we pay attention is transferred to the working/short term memory in smallest fraction of time. In other words, human brain is continuously collecting information of its relevance and processing it. This process of information acquisition starts from sensory memory and then proceeds to working memory and from there it is reflected in LTM and fetched accordingly whenever required. Figure 1, illustrates the flow of information from sensory memory to LTM.

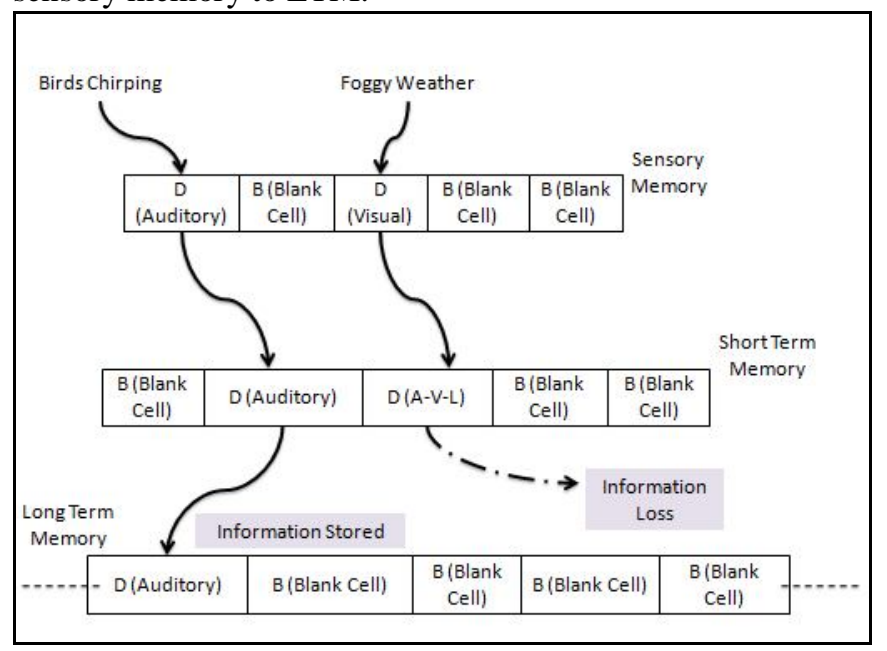

Figure 1: The flow of information from sensory to long term memory

Sensory memory takes the information and passes it to the Short Term Memory (STM). The information which is in visual form in sensory memory is converted in auditory, verbal or linguist form in STM [2]. The information on which user gave attention is passed to LTM and the information which losses attention is lost and not transmitted to LTM.

Learning and memory are directly associated to each other as knowledge is stored in a form of memory. Knowledge can be represented in three types; declarative, procedural and conditional. When we talk in terms of factual information then we are referring to our declarative knowledge. However, when we are recalling the steps for executing a process that time we are referring to our procedural knowledge. Conditional knowledge helps us in identifying which knowledge as per the scenario, whether declarative or procedural need to be applied and why. In this paper, we have 
proposed a Turing Machine (TM) model for processing information from sensory memory to working memory and working to LTM.

\section{RELATED WORKS}

Learning is one of the longest running, undeniably important actions of human being. Cognitive and emotional processes work together to improve thinking [3]. Emotions like cheerfulness, fear and mood states influence our thinking, decision making and performance. There are various perspectives by several researchers on how emotion and cognition, emotion and learning, are related to each other. According to Regan [4], learning can be enhanced by minimizing negative emotions and maximizing positive emotions.

The learning style affects the overall learning environment of a learner. The term learning style was coined in 1987 by Kefee [5] quoted as "[a learning style is] the composite of characteristic cognitive, affective and psychological factors that serve as relatively stable indicators of how a learner perceives, interacts with, and responds to the learning environment". The learning style of a learner can be categorized mainly as visual, auditory and kinesthetic. Visual learners learn through their visual stimuli, auditory learners learn through their spoken stimuli and kinesthetic learners believe in learning through their actions.

Watson, Clark and Tellegen [6] developed a Positive and Negative Affect Schedule (PANAS) scale for measuring the Positive Affect (PA) and Negative Affect (NA) of individuals. High NA indicates the individual's discomfort, unpleasantness which includes emotions like nervousness, fear and disturbance. High PA indicates the individual's well-being which includes inspiration, determination and enthusiasm. The learner's intellectual development and functioning is the focus of cognitive learning. For example, an individual attempting to acquire the skill of driving a motor vehicle should know about the different parts of the vehicle, which are directly involved in the process of driving. These aspects of learning involve cognition. But at the same time the learner's attitude towards the learning process influences the affective domain of learner.

Human perceive information from sensory organs and the information which has gained attention is procured from sensory memory and is stored in STM for further execution. The information which are well rehearsed in STM is further stored in LTM. In this way, the functionality of the memory is similar to a Turing tape [7]. Zylberberg, Dehaene, Roelfsema and Sigman [8] have proposed a TM framework for human mental programs. For instance, if we want to switch on the light then certainly our brain needs to perform certain sequential steps to execute that particular task. This sequential execution of steps depends on the concept of 'Productions'. The procedural knowledge is represented in terms of productions and scripts [9]. Production can be defined as condition-action rule, i.e. the action need to be performed in what condition. The authors have assumed that a production can be considered as an action that is equivalent to a single step of a TM.

\section{HUMAN COGNITION AND TURING MACHINE}

The knowledge acquisition and conceptual understanding includes a mental process called cognition. The never ending process of knowledge acquisition and generation in human brain can be represented in a form of multi-tape TM.

Let's consider a scenario for an instance of time:

1. Person $\mathrm{P}$ is standing in her balcony and enjoying climate

2. Listening to Chirping of Birds

3. Person $\mathrm{P}$ is reading Newspaper

4. Doorbell rings

During the scenario Person $\mathrm{P}$ is in cognition state:

1. Person $\mathrm{P}$ is having experience of this moment where she is enjoying the climate,

1.1 If she is enjoying this moment and the climate for the first time in her life then she is constructing and assimilating learning in her memory cells of brain.

Implication: The information on which Person $\mathrm{P}$ is generating interest moves from sensory stimuli to sensory memory and then from sensory to working memory, on assimilation it moves to long term memory.

1.2 But if she had earlier experience of this kind of moment then she is rehearsing on this learning again.

Implication: If Person $\mathrm{P}$ had already experienced this earlier, then the assimilated knowledge will be reflected from long term memory to person $\mathrm{P}$ working memory. Person $\mathrm{P}$ will reconstruct cognition.

Reconsider the scenario with respect to Turing machine:

1.1 The information which was attended by sensory stimuli was gathered in sensory memory cells. Each cell has capacity to store certain bits of information. This storage of cells can be assumed in form of Turing machine tape which has limited capacity to store information. The storage cell consists of only 
that information which was not lost i.e. the information which was given attention by Person $\mathrm{P}$ was stored in sensory memory and rest all was lost. For this particular instance of time information is stored and further processed in working/short term memory and from there if it is well rehearsed then it moves to LTM. Similarly, a Turing machine tape which has a finite bound capacity can be assumed for the storage of information in short term memory. LTM has infinitely long capacity for storing the data as compared to working memory and sensory memory. The learning cycle of human beings is a never ending process but it comes to an end when humans die. Thus, a finite bound Turing machine tape can be assumed for LTM also.

1.2 When person $\mathrm{P}$ had already experienced the similar kind of moment earlier, that means the information is already stored somewhere in LTM of Person P i.e. Turing machine tape of LTM. In this case the information is fetched from LTM tape to STM tape and reconstruction of knowledge takes place.

While enjoying the climate Person $\mathrm{P}$ is simultaneously sensing chirping of birds, reading newspaper from her eyes and at the same time also hears the doorbell. Thus, this can be assumed that there are multiple tapes for auditory, visual, haptic in the sensory stimuli and those information cells which have generated attention are all procured in the single sensory memory tape for further processing.

\section{A. Turing Machine Model for information transfer from Sensory to Short Term Memory}

Humans receive information through sensory organs which are stored in sensory memory. Sensory memory has limited capacity. Information is transferred from sensory to STM in a time span of 500 milliseconds. For instance, when our eyes are looking at some numbers and giving attention to them, then our brain put those numbers from sensory memory to STM. Information which is given more attention and rehearsed is then further transferred from short term to LTM. When we recall something and tries to retrieve information then the information from LTM is transferred to STM. Practically, if we look at this scenario we will find that lot of computations are being done while taking information from sensory to LTM. Similarly, in computer the data is transferred from primary memory to secondary memory and secondary to primary memory. According to Turing [10], Turing machine is capable enough to represent realistic models of computations. Random access memory consists of a finite number of registers. If we look at sensory memory same can be assumed for it. Sensory inputs are given and information is stored in sensory memory.
In this paper, a model for information transfer in memory is proposed using Turing Machine. We have assumed that we have Turing tapes each for sensory and STM. Assuming the sensory memory has cells which can store data for 500 milliseconds. One cell can contain a chunk of data and this data is read for the purpose of storing it into STM within 100 milliseconds. From sensory memory the data is sent to the STM in which there are 5 to 9 cells. Turing Tape 1 for sensory memory contains the data perceived by the sensory organs and this data can be represented as symbol ' $D$ '. The information which is not paid attention will be dropped and the cell will be left blank. The blank cell is represented by symbol ' $B$ ' and symbol ' $S$ ' represents no change in the position of tape head. Another assumption can be the limited capacity of sensory memory. For the TM model [11], we assume that the sensory memory window consists of 5 cells and the data can be read by moving the tape head in the left to right and right to left directions. The tapes are bounded by the end markers ' $\$$ ' and '\#'. The Turing tape for STM consists of some previous information that is already available and it also consists of the new information which has been transferred from the sensory to STM. The loss of information can happen at this stage also.

Thus accordingly, tape 2 will have the symbols ' $\mathrm{D}$ ' and ' $\mathrm{B}$ ' along with the case markers ' $\$$ ' and '\#'. The initial state is represented by $\mathrm{q} 0$ and both the Turing tapes heads point to $\$$. After the completion of one cycle, final state will be reached. Figure 2, shows the state transition diagram of information bits from sensory to short term memory.

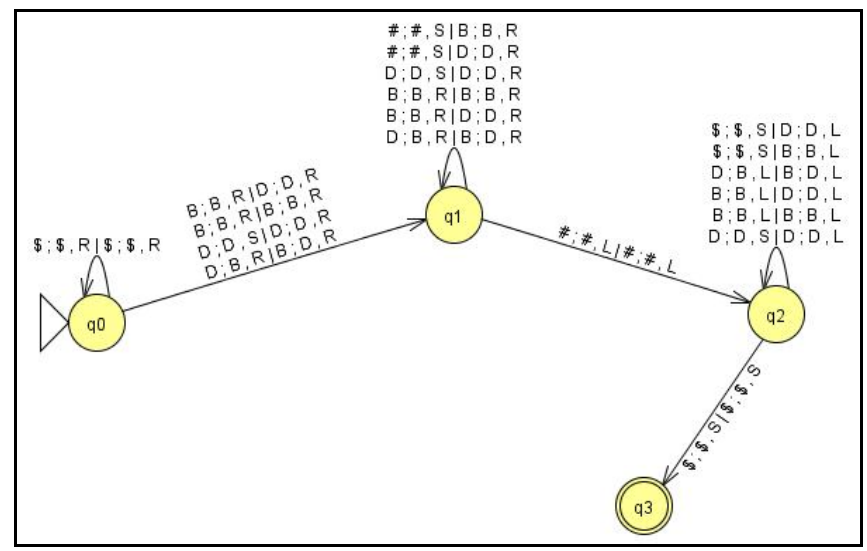

Figure 2: State transition diagram for information transfer from sensory to short term memory

The step by step movement of the head positions in figure 3(a) and 3(b) illustrates different transitions of q0, q1, q2 and q3 states. 


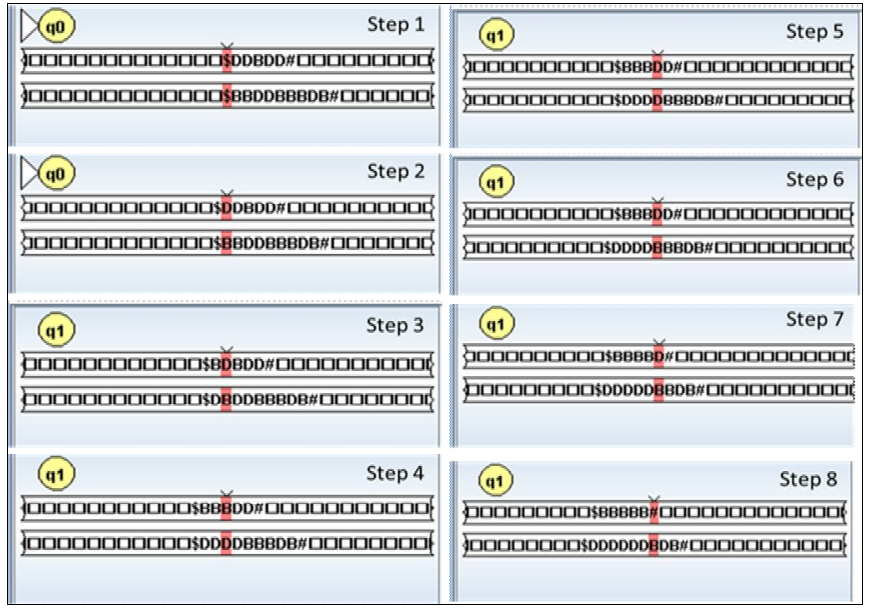

Figure 3(a): Information processing and transfer using Multi-Tape Turing machine

Tape 1 is depicting the scenario for the sensory memory and tape 2 for short term memory. Initially, both the head positions $\mathrm{H} 1$ and $\mathrm{H} 2$ of tape 1 and tape 2 respectively are pointing on $\$$ symbol. The state transition starts from qo state and moves from left to right, then right to left and during the transitions it sometimes remains at the same position. At step 5 , both the heads are on the fourth cell containing D.

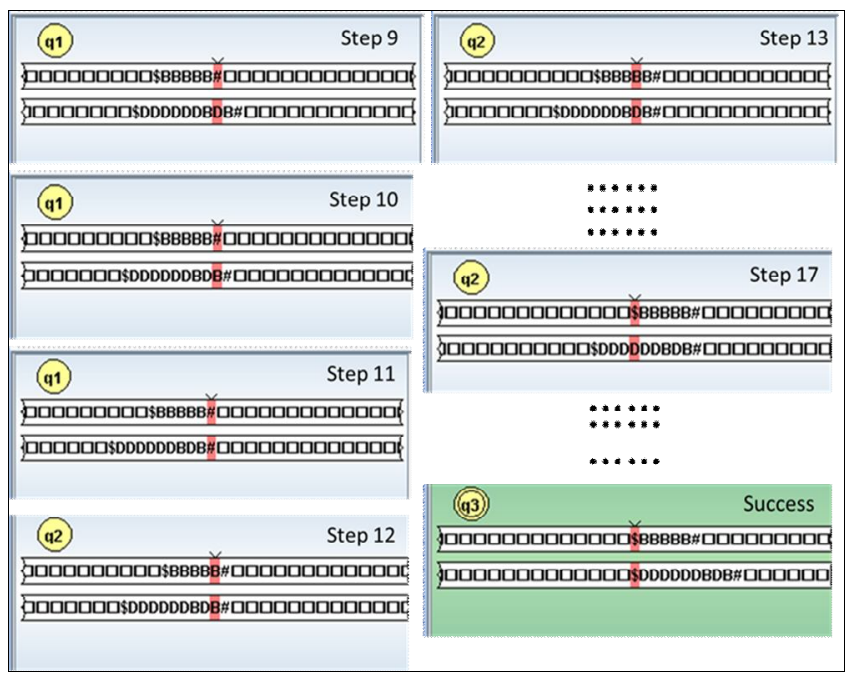

Figure 3(b): Information processing and transfer using Multi-Tape Turing machine

The machine encounters a variation at step 6 where tape 2 moves one cell ahead on the fifth cell while tape 1 head remains on the same position. Through this model, we can deduce the process of storing information in LTM and retrieving the information from LTM.

\section{DISCUSSION ON THE PROPOSED HUMAN TURING MACHINE MODEL}

Learning is an active process which occurs at LTM, but there are various factors that affect our learning process. Formulation of new knowledge depends on our previous knowledge, learning style, positive or negative aspect towards learning and the external environment. This research work simulates the working process of storing and retrieving the information in human brain by the help of multi-tape Turing machine. In the process of storing the information in human brain, some bits of information can be lost just because the learner was not giving any attention to it. In other words, the procurement of information is directly related with learner's emotional dimension, learning style and external environment.

An informal description of 3-tape Turing machine for processing information from sensory to working memory and working to LTM is depicted in figure 4.

Assuming that the input has the form \$BBDDD\# where D can be the information in any sensory stimuli form and given on the first tape, we can call it sensory tape. The information need to be transmitted on the second tape called STM tape and the third tape called LTM tape. Sensory and STM are finite memory.

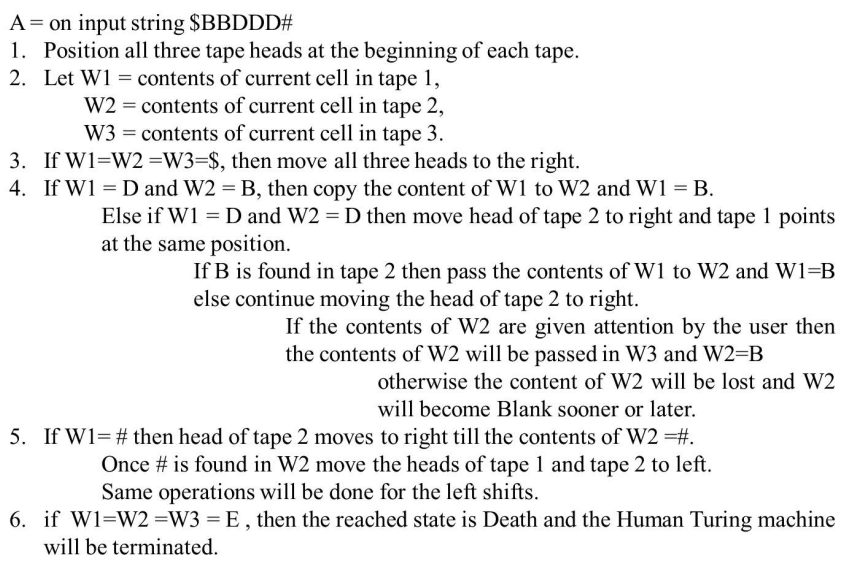

6. if $\mathrm{W} 1=\mathrm{W} 2=\mathrm{W} 3=\mathrm{E}$, then the reached state is Death and the Human Turing machine will be terminated.

Figure 4: Informal Description of 3-Tape Human TM

If we consider that a human memory starts working when a child is born and ends when the person dies then this assumption makes the LTM as finite memory. Here the initial state can be the born state and the final state will be the death represented as ' $\mathrm{E}$ '.

\section{CONCLUSION}

In this research paper, a model for the transfer and retrieval of the information from sensory memory to LTM has been demonstrated using multi-tape TM. Learning is reflected when the knowledge is constructed in LTM. However, LTM comprise of only those knowledge which has exhibited learner's interest. Thus, it is essential to understand that what is the emotional dimension of learner, how, and in which style, learner wants to learn the information. 


\section{REFERENCES}

1. J. V. Wertsch. From Social Interaction to Higher Psychological Processes a Clarification and Application of Vygotsky's Theory, Human Development, vol. 22, no. 1, pp. 1-22, 1979.

2. R. Atkinson and R. Shiffrin. Human Memory: A Proposed System and its Control Processes, Psychology of Learning and Motivation, pp. 89-195, 1968.

3. M. A. Brackett, S. E. Rivers, and P. Salovey. Emotional Intelligence: Implications for Personal, Social, Academic, and Workplace Success, Social and Personality Psychology Compass, vol. 5, no. 1, pp. 88-103, 2011.

4. K. O'Regan. Emotion and E-Learning, Online Learning, vol. 7, no. 3, 2019.

5. J. W. Keefe. Learning Style Theory and Practice, National Association of Secondary School Principals, ERIC, 1987.

6. D. Watson, L. A. Clark, and A. Tellegen. Development and validation of brief measures of positive and negative affect: The PANAS scales, Journal of Personality and Social Psychology, vol. 54, no. 6, pp. 1063-1070, 1988.

7. A. J. Wells. Cognitive Science and the Turing Machine: an Ecological Perspective, Alan Turing: Life and Legacy of a Great Thinker, pp. 271-292, 2004.

8. A. Zylberberg, S. Dehaene, P. R. Roelfsema, and M. Sigman. The human Turing machine: a neural framework for mental programs, Trends in Cognitive Sciences, 2011.

9. J. R. Anderson, J. M. Fincham, Y. Qin, and A. Stocco. A central circuit of the mind, Trends in Cognitive Sciences, vol. 12, no. 4, pp. 136-143, 2008.

10. A. M. Turing. On Computable Numbers, with an Application to the Entscheidungsproblem, Proceedings of the London Mathematical Society, vol. s2-42, no. 1, pp. 230-265, 1937.

11. S. Rodger. Learning automata and formal languages interactively with JFLAP, ACM SIGCSE Bulletin, vol. 38 , no. 3 , p. $360,2006$. 